\title{
Magnetic nanographite
}

\author{
Koichi Kusakabe ${ }^{\text {f }}$ and Masanori Maruyama f \\ Graduate School of Science and Technology, Niigata University, \\ Ikarashi, Niigata 950-2181, Japan
}

(Dated: November 1, 2018)

\begin{abstract}
Hydrogenated nanographite can display spontaneous magnetism. Recently we proposed that hydrogenation of nanographite is able to induce finite magnetization. We have performed theoretical investigation of a graphene ribbon in which each carbon is bonded to two hydrogen atoms at one edge and to a single hydrogen atom at another edge. Application of the local-spin-density approximation to the calculation of the electronic band-structure of the ribbon shows appearance of a spin-polarized flat band at the Fermi energy. Producing different numbers of mono-hydrogenated carbons and dihydrogenated carbons can create magnetic moments in nanographite.

PACS numbers: 75.75.+a, 75.50.Xx, 73.20.At, 73.22.-f
\end{abstract}

Recent reports on magnetic carbon have stimulated renewed interest in carbon systems as possible new magnetic materials made exclusively of light elements. These investigations include experiments suggesting ferromagnetic behavior in polymerized fullerenes made using photo-assisted oxidation [1] or high-pressure treatment. [2] A nanometer-scale graphite called nanographite may yield another magnetic carbon system. Some experiments have demonstrated the existence of anomalous magnetic behavior unexpected in diamagnetic bulk graphite.[3, . . 5]

Several theoretical investigations of nanographite have been carried out prior to or parallel to these experiments. 6, 6, 8, 9, 10, 11, 12, 13, 14 An important finding was that $\pi$ electrons in nanometer-scale graphite are strongly affected by structure of edges. A typical edge is the zigzag edge. The zigzag edge was found in STM images of nanometer scale graphene, [15, 16] although the armchair edge has lower energy than the zigzag edge. 17, 18] Fujita and coworkers suggested that the $\pi$ electrons on a mono-hydrogenated zigzag edge may create a ferrimagnetic spin structure on the edge. [6] Magnetism with localized spin moments is possible due to the existence of non-bonding localized states at the zigzag edge. These states are called edge states and are highly degenerate at the Fermi level and may be spin polarized. Such an edge state does not appear on an armchair edge. Klein has studied another edge of graphene strips, considering a zigzag edge with an atomic site having a $\pi$-orbital on the edge.[19, 20] Examining a tight-binding model of $\pi$ electrons, he found degenerate surface states on the edge, which is referred to here as a bearded edge.

The total spin moment of a graphene ribbon, however, should be zero, if both of the two edges of the ribbon are zigzag edges, or if both of them are bearded edges. This is because, the structure of the $\pi$ network becomes a bipartite lattice having the same number of sublattice sites. In

\footnotetext{
*Electronic address: kabe@sunshine.gs.niigata-u.ac.ip

${ }^{\dagger}$ Electronic address: maruyama@sunshine.gs.niigata-u.ac.jp
}

such cases, an argument using the Hubbard model results in zero spin moment, following the Lieb theorem. [6, 21] Naively, we may say that local magnetic moments at the two edges are coupled anti-ferromagnetically. [6]

In this paper, we show that a hydrogenated graphene ribbon can have a finite total magnetic moment. A key point is the structure of the two edges of the ribbon. In our ribbon, one of the edges is composed of monohydrogenated carbon atoms and another is made of dihydrogenated ones. By a first-principles electronic-state calculation, we will show that the system is stable and has a fully spin-polarized flat band. A finite spontaneous magnetization appears in the graphene ribbon.

We present here our idea to find magnetic nanographite. If a graphene ribbon has a bearded edge on one side and a zigzag edge on the other side, the $\pi$ network becomes a bipartite lattice with different numbers of sublattice sites.[22, 23] Then, a flat band appears at the Fermi level. The band is fully spin polarized if inter-electron interactions e.g. the Hubbard repulsion $U$ is considered. 21] In addition, the flat band consists of edge states, so that the magnetic moments are localized at both edges and the distribution of moments shows exponential decay in the $\pi$ network. 23. However, to design a realistic material, we also have to specify the atomic configuration of the bearded edge.

We found that one solution is a zigzag edge whose end carbon atoms are di-hydrogenated.24 In order to present an example of magnetic nanographite, we will show the electronic band structure of the ribbon which has a completely spin-polarized flat band at the Fermi energy. The magnetism will be explained using a Hubbard model.

To investigate the stability and magnetic structure of the nanographite, we used an electronic-state calculation with a local spin-density approximation (LSDA) based on the density functional theory. 225, 26] An LSDA functional given by Perdew and Wang 27 was utilized as the exchange-correlation energy functional. The ultra-soft pseudopotential generated using the Vanderbilt strategy was adopted. 28. The valence wave functions were expanded in a plane-wave (PW) basis set. To achieve con- 
vergence in the total energy, we used an energy cut-off $E_{c}$ of 49Ry for structural optimization in a fixed unit cell. To reduce computation time, we adopted $E_{c}=25 \mathrm{Ry}$ to optimize the size of the unit cell before the final optimization of inner coordinates. Structural optimization was performed until each component of the interatomic force became less than $8 \times 10^{-5} \mathrm{H} / \mathrm{a}$.u. The calculations were done using a computer program called the Tokyo ab-initio program package (TAPP). 29

To check the accuracy of our calculation, we obtained the structural parameters of a hydrogen molecule, a $\mathrm{CH}_{4}$ molecule, a $\mathrm{C}_{2} \mathrm{H}_{4}$ molecule, diamond and graphite. The bond length of each molecule and the bond angles of $\mathrm{CH}_{4}$ and $\mathrm{C}_{2} \mathrm{H}_{4}$ were obtained with relative errors less than $3.3 \%$. The vibrational energy $\hbar \omega_{e}$ of $\mathrm{H}_{2}$ was $1.92 \times 10^{-2}$ $[\mathrm{H}]$, which is about $4 \%$ smaller than $2.00 \times 10^{-2}[\mathrm{H}]$ observed. For cubic diamond, the lattice constant, the bulk modulus and its pressure derivative were 6.677 a.u., 4.66 Mbar and 3.68. For hexagonal graphite, the lattice constants were $a=4.616$ a.u. and $c=12.67$ a.u. These values are in reasonable agreement with the values determined experimentally or by previous calculations. 30 Band structures of the solids also reproduced previous results. 30.

Consider the graphene ribbon shown in Fig. 1. In this structure, each dark circle represents the position of a carbon atom. Solid lines represent $\sigma$ bonds. Each open circle is a hydrogen atom. For convenience, we number atoms and name them $\mathrm{C}^{(1)} \ldots \mathrm{C}^{(2 n)}$ and $\mathrm{H}^{(1)} \ldots \mathrm{H}^{(3)}$ in a unit cell. Here, $n$ is a positive integer. On the left edge, each carbon atom is bonded to a hydrogen atom and has a $\pi$ orbital. On the right edge, each carbon atom has bonds to two hydrogen atoms and two neighboring carbon atoms. If we consider that the di-hidrogenated carbon atom $\mathrm{C}^{(2 n)}$ forms $\mathrm{sp}^{3}$ bonds instead of $\mathrm{sp}^{2}$ bonds, $\mathrm{C}^{(2 n)}$ loses a $\pi$ electron. Since there remain $2 n-1$ carbon atoms having a $\pi$ electron per each in a unit cell, the $\pi$ network might become a bipartite lattice with different numbers of sublattice sites.

We can consider ribbons with various widths. However, we know that the optimum width is given by $n \simeq 5 \sim 10$ for nanographite ribbons with zigzag edges. [7] In any case, the essential properties should be the same irrespective of the width. Thus we first consider a ribbon with $n=5$ and discuss dependence of magnetism on the width of the graphene ribbon at the end of the paper.

To examine our idea, we have performed an LSDA calculation. Below, we assume a periodic boundary condition. Since the structure is one-dimensional, we are allowed to use $k$ points along the $y$ axis as $\left(0, k_{y}, 0\right)$ for the structural optimization. We used $6 k$ points uniformly distributed in the Brillouin zone for most simulations. We determined the equilibrium lattice constant of the ribbon, which became 4.674 a.u. For the calculation to optimize the inner parameters, the size of the cell was taken to be $40.27 \times 4.647 \times 18.60$ (a.u. $)^{3}$. The spacing between neighboring ribbons became 19.5 a.u. in the $x$ direction and 15.3 a.u. in the $z$ direction. The structure

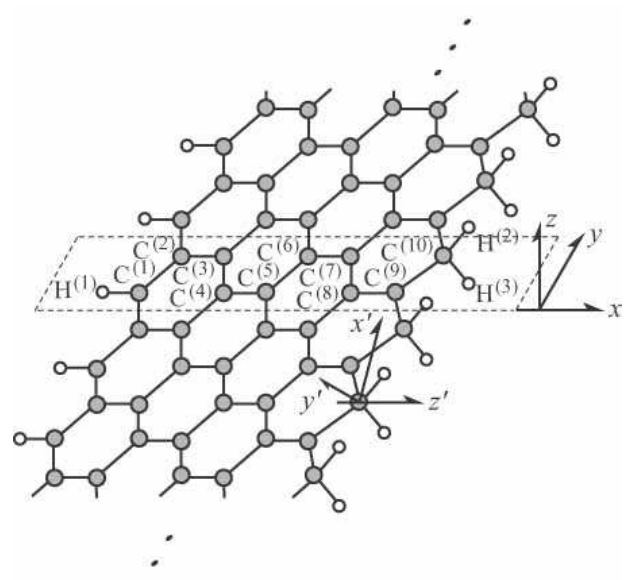

FIG. 1: A structural model of a magnetic graphene ribbon. Dark circles represent carbon atoms and open circles denote hydrogen atoms. Dashed lines represent a section of a unit cell in the $x y$-plane. The unit cell contains $2 n$ carbon atoms and 3 hydrogen atoms. Here a case with $n=5$ is shown. Two sets of 3-dimensional coordinate axes, $(x y z)$ and $\left(x^{\prime} y^{\prime} z^{\prime}\right)$, are displayed. The $x$-axis and the $y$-axis are in a graphene plane. Three axes $x^{\prime}, y^{\prime}$ and $z^{\prime}$ point to the (011), (0-11), (100) directions, respectively, in the $(x y z)$ coordinate.

was optimized by a conjugate gradient method. The obtained results did not change when we used $12 k$ points instead. The bond length of C-C ranged from about 2.64 $\sim 2.70$ a.u. for neighboring carbon pairs except for 2.59 a.u. between $\mathrm{C}^{(8)}$ and $\mathrm{C}^{(9)}$ and 2.76 a.u. between $\mathrm{C}^{(9)}$ and $\mathrm{C}^{(10)}$. Bond angles $\mathrm{H}^{(2)}-\mathrm{C}^{(10)}-\mathrm{H}^{(3)}$ and $\mathrm{C}^{(9)}-\mathrm{C}^{(10)}$ $\mathrm{C}^{(9)}$ are $100.7^{\circ}$ and $114.8^{\circ}$. These structure parameters suggest that the end carbon $\mathrm{C}^{(10)}$ forms $\mathrm{sp}^{3}$ bonds.

An electronic band structure of the graphene ribbon is shown in Fig. 2. In the first Brillouin zone, the $\Gamma-Y$ line corresponds to a one-dimensional axis on which a $k$ vector is parallel to the ribbon. A flat band appears at the Fermi level. The band is almost dispersionless in the whole Brillouin zone and is completely spin polarized. That is, only the spin-up branch is filled and the spin-down branch is empty. Splitting between polarized flat bands for up-electrons and down-electrons is approximately $0.5 \sim 0.6 \mathrm{eV}$ and the gap between them is $\sim 0.2$ $\mathrm{eV}$. These values give a rough energy scale of stiffness for the magnetic ground state. The total spin $S_{t o t}$ becomes $1 / 2$ times number of unit cells $N_{c}$. Stability of the polarized flat band was confirmed by using a unit cell which was doubled in the $y$ direction.

The flat band is the center band of 9 well-characterized $\pi$-bands. See band dispersion around the $Y$ point in Fig. 2. The dispersion relation of these $\pi$ bands is roughly the same as those obtained for a simple tight binding model with $9 \pi$-orbitals on a graphene ribbon. In Fig. 2, all branches for up-electrons and down-electrons are split by spin polarization. This result implies that a magnetic moment appears on every atom. We show obtained spin density in Fig. 3. A ferrimagnetic spin structure is clearly 


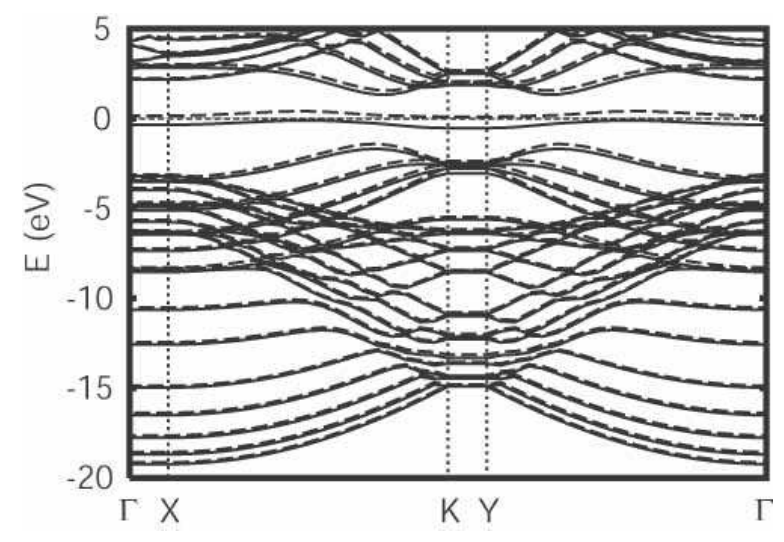

FIG. 2: The electronic band structure of a hydrogenated graphene ribbon. Solid lines and dashed lines represent the dispersion relation of bands for up electrons and down electrons, respectively. At the Fermi level $E_{F}=0$, a dispersionless band appears. The band is completely spin polarized. Above and blow the flat band, there are $8 \pi$-bands, which are from the 18 th band to the 21 th band and from the 23 th band to the 26 th band on the $K-Y$ line. The 17 th band on the $K-Y$ line is composed mainly of 1 s orbitals of two hydrogen atoms $\mathrm{H}^{(2)}$ and $\mathrm{H}^{(3)}$ in Fig. 1.

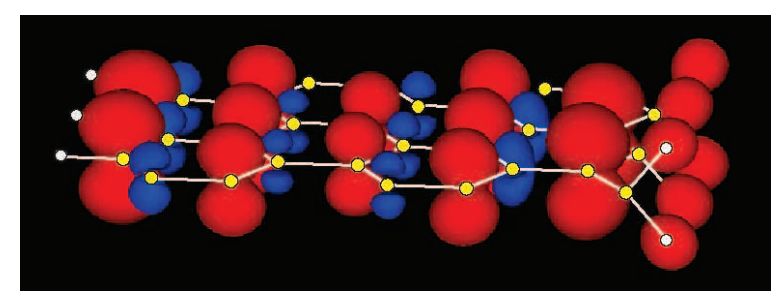

FIG. 3: The optimized structure of a magnetic graphene ribbon. Yellow spheres and white spheres represent the positions of carbon atoms and hydrogen atoms, respectively. Spin density on the ribbon is displayed by isosurfaces. Red surfaces and blue surfaces represent spin-up density and spin-down density. The isolevel is set to $1 / 4$ of the maximum amplitude of the spin-up density.

seen. A local spin moment exists on every carbon atom. Interestingly, finite spin moments appear on two hydrogen atoms which have bondings with $\mathrm{C}^{(10)}$. The reason will be explained below. Thus, the graphene ribbon is confirmed to be magnetic in the LSDA model. We call this structure magnetic nanographite.

We found a curious characteristic of the wave functions of the flat band. We surmise that only 9 carbon atoms in the unit cell possess $\pi$ orbitals which contribute to the $\pi$ bands. This is because $\mathrm{sp}^{3}$ bonds are created at the dihydrogenated carbon atom $\mathrm{C}^{(10)}$. Wave functions on the flat band were non-bonding edge states with finite amplitude on only odd-numbered carbon atoms, but not on even-numbered ones. An interesting point is that a finite amplitude was found on two hydrogen atoms $\mathrm{H}^{(2)}$ and $\mathrm{H}^{(3)}$. Therefore, we have to consider 1s orbitals on these hydrogen atoms in order to analyze the spin-polarized flat band.

Let us introduce two sets of orthogonal coordinates, $(x y z)$ and $\left(x^{\prime} y^{\prime} z^{\prime}\right)$ as defined in Fig. 11. Four $\mathrm{sp}^{3}$ orbitals on $\mathrm{C}^{(10)}$ may be represented as $\phi_{1}=\phi_{s}+\phi_{x^{\prime}}+\phi_{y^{\prime}}+\phi_{z^{\prime}}$, $\phi_{2}=\phi_{s}-\phi_{x^{\prime}}-\phi_{y^{\prime}}+\phi_{z^{\prime}}, \phi_{3}=\phi_{s}+\phi_{x^{\prime}}-\phi_{y^{\prime}}-\phi_{z^{\prime}}$, and $\phi_{4}=\phi_{s}-\phi_{x^{\prime}}+\phi_{y^{\prime}}-\phi_{z^{\prime}}$. Here, $\phi_{s}$ is a 2s orbital and $\phi_{m}\left(m=x^{\prime}, y^{\prime}, z^{\prime}\right)$ are three 2 p orbitals on $\mathrm{C}^{(10)}$. $\phi_{1}$ and $\phi_{2}$ point to $\mathrm{H}^{(2)}$ and $\mathrm{H}^{(3)}$, respectively. We take linear combinations of $\phi_{1}$ and $\phi_{2}$ as well as 1 s orbitals $\varphi_{1}$ and $\varphi_{2}$ on $\mathrm{H}^{(2)}$ and $\mathrm{H}^{(3)}$ and define $\bar{\phi}_{ \pm}=\left(\phi_{1} \pm \phi_{2}\right) / \sqrt{2}$ and $\bar{\varphi}_{ \pm}=\left(\varphi_{1} \pm \varphi_{2}\right) / \sqrt{2}$. Note that $\bar{\phi}_{-}=\phi_{z}$, i.e. a $\pi$ orbital on $\mathrm{C}^{(10)}$. By symmetry, $\bar{\phi}_{-}$and $\bar{\varphi}_{-}$hybridize with the $\pi$ orbitals of the host $\pi$ system of the graphene sheet, but not with $\sigma$ orbitals in the honeycomb lattice. Therefore, we must utilize $\bar{\phi}_{-}$and $\bar{\varphi}_{-}$as well as the $\pi$ orbitals on $\mathrm{C}^{(i)}(i=1, \cdots 9)$ to analyze the $\pi$ network in the magnetic nanographite.

If we adopt the above representation, we obtain an effective model describing the ribbon. The model consists of 11 local orbitals in a unit cell. Here we ignore overlap integrals between orbitals for simplicity. Introducing the operator representation $c_{i, \sigma}$ for a $\pi$ electron on a carbon atom and $d_{l, \sigma}$ for an electron in $\bar{\varphi}_{-}$in the $l$-th unit cell, we obtain an effective Hamiltonian.

$$
\begin{aligned}
H= & -\sum_{\langle i, j\rangle} \sum_{\sigma} t_{i, j}\left(c_{i, \sigma}^{\dagger} c_{j, \sigma}+H . c .\right)+\sum_{i} U n_{i, \uparrow} n_{i, \downarrow} \\
& -\sum_{\langle i, l\rangle} \sum_{\sigma} t_{i, l}^{\prime}\left(c_{i, \sigma}^{\dagger} d_{l, \sigma}+H . c .\right)+\sum_{l} U^{\prime} m_{l, \uparrow} m_{l, \downarrow} \\
& +\varepsilon \sum_{l} \sum_{\sigma} m_{l, \sigma}
\end{aligned}
$$

Here, an index $i$ specifying a carbon atom runs over all carbon sites. Two kinds of number operators are defined as $n_{i, \sigma} \equiv c_{i, \sigma}^{\dagger} c_{i, \sigma}$ and $m_{l, \sigma} \equiv d_{l, \sigma}^{\dagger} d_{l, \sigma}$. Bond connections given by non-zero $t_{i, j}$ and $t_{i, l}^{\prime}$ are the same as those in a graphene ribbon with a zigzag edge and a bearded edge.23 We set the origin of the energy at the orbital energy of the $2 \mathrm{p}$ orbital $\varepsilon_{2 p}$ on carbon. $\varepsilon_{1 s}$ denotes the energy of a 1 s electron on a hydrogen atom and $\varepsilon$ is given by $\varepsilon=\varepsilon_{1 s}-\varepsilon_{2 p}$. Since $\varepsilon<0$, a band mainly composed of $\bar{\varphi}-$ is separated in energy from other $\pi$ bands. This property is also seen in the LSDA band structure, where the 17th band on the $K-Y$ line in Fig. 2 is the corresponding band.

Consider a periodic boundary condition for a finite system with $N_{c}$ unit cells. If $\varepsilon=0$ and if $U^{\prime}=U$, Lieb's theorem is applicable and the ground state becomes a ferrimagnetic state with a total magnetization $S_{\text {tot }}=\left|N_{A}-N_{B}\right| / 2$. Here the difference in sublattice sites $\left|N_{A}-N_{B}\right|$ equals $N_{c}$. This magnetic ground state is thought to be stable against perturbation like $\varepsilon<0$, since 1) there is a finite gap between the flat band and other $\pi$ bands both in the tight-binding description and in the LSDA result in Fig. 2, 2) we have confirmed that a small difference of $\varepsilon_{1 s}$ and $\varepsilon_{2 p}$ does not break the flatness of the center band in the tight-binding model. Thus the 
magnetism found in an LSDA calculation can be understood as the appearance of ferrimagnetism in a flat-band Hubbard model.

The formation energy of a di-hydrogenated edge has been evaluated by comparing the total energy within LSDA. We denote $E_{H_{2}}, E_{G 1}$ and $E_{G 2}$ as the energy of a hydrogen molecule, that of a graphene ribbon with mono-hydrogenated zigzag edges and that of a magnetic nanographite ribbon, respectively. The latter two are energy per a unit cell including $2 n$ carbon atoms. Our calculation shows that $E_{G 2}-\left(E_{G 1}+E_{H_{2}} / 2\right) \simeq 0.3 \mathrm{eV}$ for $n=5$. Thus, the hydrogenation of nanographite with mono-hydrogenated zigzag edges is confirmed to be an endothermic reaction. Utilization of proper catalysts in catalytic reduction may be important in the synthesis of magnetic nanographite.

To see how our conclusion is affected by changing the width of the ribbon, we performed the LSDA calculation for ribbons with a width from $n=2$ to $n=4$. A ferrimagnetic structure was found even in these narrow ribbons. These ribbons do not show the Peierls distortion, which is similar to conclusions on the mono-hydrogenated zigzag ribbons. 8, 10, 31

We have shown that a spin-polarized flat band appears in the LSDA band structure of a graphene ribbon. The origin of the magnetism is explained as a realization of the flat-band ferromagnetism. An orbital $\bar{\varphi}$ - on two hydrogen atoms $\mathrm{H}^{(2)}$ and $\mathrm{H}^{(3)}$ is an anti-bonding orbital and has symmetry $p_{z}$. Thus, hydrogenation of a carbon atom is identified as the addition of a $\pi$ orbital at a zigzag edge. The structure may be regarded as a realization of the bearded edge. We can expect magnetism for any hydrogenated ribbon with $\left|N_{A}-N_{B}\right|>0$, even if di-hydrogenation of the zigzag edge is not perfect. The same effect is also expected with fluorination. 32 In this report we have considered a ribbon with a flat graphene structure, but the method is also applicable to nanotubes with zigzag edges. Thus it appears that, a magnetic nanotube may be designed. 24.

\section{Acknowledgments}

One of the authors K.K. is grateful for fruitful discussions with Professor T. Enoki and Dr Takai. This work has been supported by a Grant-in-Aid from the Ministry of Education, Culture, Sports, Science and Technology of Japan. The calculation was partly performed at the computer facility of ISSP, University of Tokyo.
[1] Y. Murakami and H. Suematsu, Pure Appl. Chem. 68, 1463 (1996).

[2] T. L. Makarova, B. Sundqvist, R. Hohne, P. Esquinazi, Y. Kopelevich, P. Scharff, V. A. Davydov, L. S. Kashevarova, and A. V. Rakhmanina, Nature 413, 716 (2001).

[3] Y. Shibayama, H. Sato, T. Enoki, and M. Endo, Phys. Rev. Lett. 84, 1744 (2000).

[4] B. L. V. Prasad, H. Sato, T. Enoki, Y. Hishiyama, Y. Kaburagi, A. M. Rao, P. C. Eklund, K. Oshida, and M. Endo, Phys. Rev. B 62, 11209 (2000).

[5] Y. Kopelevich, P. Esquinazi, J. H. S. Torres, and S. Moehlecke, J. Low Temp. Phys. 119, 691 (2000).

[6] M. Fujita, K. Wakabayashi, K. Nakada, and K. Kusakabe, J. Phys. Soc. Jpn. 65, 1920 (1996).

[7] K. Nakada, M. Fujita, G. Dresselhaus, and M. Dresselhaus, Phys. Rev. B 54, 17954 (1996).

[8] M. Fujita, M. Igami, and K. Nakada, J. Phys. Soc. Jpn. 66, 1864 (1997).

[9] K. Wakabayashi, M. Sigrist, and M. Fujita, J. Phys. Soc. Jpn. 67, 2089 (1998).

[10] Y. Miyamoto, K. Nakada, and M. Fujita, Phys. Rev. B 59, 9858 (1999).

[11] K. Harigaya, J. Phys. Condens. Matter 13, 1295 (2001).

[12] K. Harigaya, Chem. Phys. Lett. 340, 123 (2001).

[13] S. Okada and A. Oshiyama, Phys. Rev. Lett. 87, 146803 (2001).

[14] K. Harigaya and T. Enoki, Chem. Phys. Lett. 351, 128 (2002).

[15] T. Land, T. Micherly, R. Behm, J. Hemminger, and G. Comsa, Surf. Sci. 264, 261 (1992).

[16] A. Nagashima, H. Itoh, T. Ichikawa, C. Oshima, and
S. Otani, Phys. Rev. B 50, 4756 (1994).

[17] A. Thess, R. LEe, P. Nikolaev, H. Dai, P. Petit, J. Robert, C. Xu, Y. Lee, S. Kim, A. Rinzler, et al., Science 273, 483 (1996).

[18] Y. Lee, S. Kim, and D. Tománek, Phys. Rev. Lett. 78, 2393 (1997).

[19] D. J. Klein, Chem. Phys. Lett. 217, 261 (1994).

[20] D. J. Klein and L. Bytautas, J. Phys. Chem. A 103, 5196 (1999).

[21] E. Lieb, Phys. Rev. Lett. 64, 1201 (1989).

[22] K. Wakabayashi and M. Sigrist, Phys. Rev. Lett. 84, 3390 (2000).

[23] K. Kusakabe and Y. Takagi, to appear in Mol. Cryst. Liq. Cryst. (2002).

[24] K. Kusakabe and M. Maruyama, to appear in TANSO (in Japanese) (2002).

[25] P. Hohenberg and W. Kohn, Phys. Rev. 136, B864 (1964).

[26] W. Kohn and L. Sham, Phys. Rev. 140, A1133 (1965).

[27] J. P. Perdew and Y. Wang, Phys. Rev. B 45, 13244 (1992).

[28] D. Vanderbilt, Phys. Rev. B 41, 7892 (1990).

[29] J. Yamauchi, M. Tsukada, S. Watanabe, and O. Sugino, Phys. Rev. B 54, 5586 (1996).

[30] J. Furthmuller, J. Hafner, and G. Kresse, Phys. Rev. B 50, 15606 (1994).

[31] M. Kertesz and R. Hoffmann, Solid State Commun. 47, 97 (1983).

[32] M. Maruyama and K. Kusakabe, unpublished (2002). 